\title{
Analytic models of electron impact excitation cross sections*
}

\author{
A. E. S. Greter \\ Department of Physics and Astronomy, University of Floride, Gainesville \\ Florida 32601, U.S.A. \\ and \\ R. S. Stolarski \\ Space Physics Research Laboratory, Department of Electrical Engineering \\ University of Michigan, Ann Arbor, Michigan 48105, U.S.A.
}

(Received 25 April 1972)

\begin{abstract}
We present a number of analytic forms which have been used to represent electron impact excitation cross sections all the way from threshold to the high energy domain where they join approximately to the results of the Born-Bethe approximation. Techniques for estimating the parameters in these analytic forms are described in detail so that the reader can update his own parameter set when new experimental information becomes available. A short collection of parameters for excitations to key states of $\mathrm{N}_{2}, \mathrm{O}_{2}$ and $\mathrm{O}$ is given along with references to sources where more complete sets are available. The importance of analytic models as a means of communication to aeronomical users of cross sections is discussed.
\end{abstract}

\section{INTRODUOTION}

THE OLOSH dependence of ionospheric physics upon atomic and molecular physics is widely recognized and as the field of ionospheric physics progresses, it becomes increasingly necessary to make use of detailed atomic properties. Many of these properties are still uncertain both from an experimental and a theoretical viewpoint. Under these circumstances analytic representations based upon phenomenological studies can serve a useful role as a convenient way of 'inputting' the latest estimate of atomic properties into calculations. The present paper is concerned with a disoussion of species which are of major importance to ionospheric calculations and with simple ways of assigning approximate electron impact cross sections.

The most important species in the Earth's ionosphere are $\mathrm{N}_{2}, \mathrm{O}_{2}$ and $\mathrm{O}$. Since the data on these species is still in the rapid accumulation stage, it is impossible at this time to make definitive assignments of all cross sections. In the case of $\mathbf{N}_{2}$ and $\mathbf{O}_{2}$, there has been a considerable recent accumulation of data. In the case of atomio oxygen, apart from the low lying states, relatively little data or analyses are available since our previous attempt at systematizing these cross sections. Accordingly, we shall concern ourselves primarily with a modest updating of earlier works, with rendering their results more easily accessible and with presenting a 'do-it-yourself kit' which would enable any investigator to parametrize new data or a proposed theoretical result in one or more convenient analytic ways.

The choice of a convenient analytic form of $\sigma_{j}(E)$, the inelastic cross section for exciting the $j^{\text {th }}$ stato as a funotion of tho incident cncrgy $(E)$, to somo oxtent doponds upon the detailed use that will be made of it. The analytic cross section may simply serve to provide a smooth approximate input for computer calculations, which is

* Supported in part by grants from the National Aeronautics and Space Administration. 
frequently advantageous compared to using a table of data values which may involve unreal discontinuities due to experimental errors. In this case, a varicty of analytic forms can be used, since differences of form are only of small consequence to a computer. For such a purpose the best function would usually be the one which most precisely represents the experimental data.

In many instances it is very advantageous to have the analytic form $\sigma(E)$ be compatible with another analytic form in an integral which, for utility, should come out to be a third analytic form. Such requirements impose sharp restrictions as to useful analytic forms. For example, in computing reaction rates, one mathematically folds the cross section with Boltzmann function. Here, for $\sigma(E)$, one wants an analytic function which has a convenient Laplace transform to obtain a simple analytic reaction rate. In many aeronomical calculations the need arises to fold a cross section into the reciprocal of a total loss function. Such loss functions can be represented analytically over a broad energy range since they are generally quite smooth (above say $25 \mathrm{eV}$ ). Here the analytic forms used to represent the loss function must be designed to go together with the form chosen for the cross section and vice versa. In this paper we shall assemble a condensed set of analytic cross sections for $\mathrm{N}_{2}, \mathrm{O}_{2}$ and $\mathrm{O}$ which largely are based upon earlier examinations of the experimental data. The cross sections are described by analytic functions of the form

$$
\sigma(E)=\left(q_{0} A / W^{2}\right) \varepsilon^{-\Omega} \Phi_{I}(\varepsilon)
$$

where $q_{0}=4 \pi a_{0}{ }^{2} R^{2}=6.514 \times 10^{-14} \mathrm{eV}^{2} \mathrm{~cm}^{2}, a_{0}$ is the Bohr radius, $R$ is the Rydberg energy, $W$ is a threshold excitation energy, $\varepsilon=E / W$, and $\Omega, \nu$ and $A$ are fitting parameters. In the form used by GREEN and BARTH (1965)

$$
\Phi_{I}=\left[1-\varepsilon^{-1}\right]^{\nu} .
$$

Since we cannot describe all variants of cross sections, our plan in this paper is to indicate an approach to such techniques and to provide a 'do-it-yourself' kit whereby an investigator can quickly and simply explore a number of alternative analytic representations and choose one which fits the data and at the same time is convenient to use. In this way the reader himself can update the parameters of various cross sections as new data comes in or as contradictions between various sources of old data are resolved.

\section{On Curve Fitting Cross Shction Data}

In the case of excitation cross sections, various analytic forms proposed generally try to achieve asymptotic agreement with the results of the Born-Bethe approximation (BBA). For allowed and/or various degrees of forbidden transitions at high energies, these go to $\varepsilon^{-1} \ln \varepsilon, \varepsilon^{-1}, \varepsilon^{-2}$ and $\varepsilon^{-3}$ (MOTT and MAssEy, 1965). In laboratory studies, the allowed transitions usually assume the most important role. However, forbidden transitions often play a very important role in aeronomical problems.

The simple power dependences of the forbidden transition are convenient for many analytic manipulations, but the logarithmic term in the allowed case presents mathematical difficulties which forces one to numerical integrations. One can unify all asymptotic excitation cross sections into the common form $\varepsilon^{-\Omega}$. By taking advantage of the fact that over an important range of $\varepsilon, \ln \varepsilon \sim \varepsilon^{\omega}$ where 
$\omega \sim \frac{1}{\text {. }}$. Specifically, we may represent various relationships which go over to $\varepsilon$ in forms such as

$$
\ln \varepsilon=R_{0} \sqrt{2} \varepsilon^{1 / 4}, \text { or } \ln (\varepsilon+1)=R_{1} \sqrt{2} \varepsilon^{1 / 4}
$$

or

$$
\ln (\varepsilon+e)=R_{\mathrm{e}} \sqrt{2} \varepsilon^{1 / 4}
$$

where the ratios $(R)$ are very close to unity. These relationships are illustrated in Fig. 1 where the three ratios are shown on log-log paper (with an expanded vertical scale). Also shown is $\varepsilon^{-1}$ to illustrate how slowly varying these ratios are. $R_{\theta}$ in particular deviates very little from unity over the range $0.2<\varepsilon<500$. We see

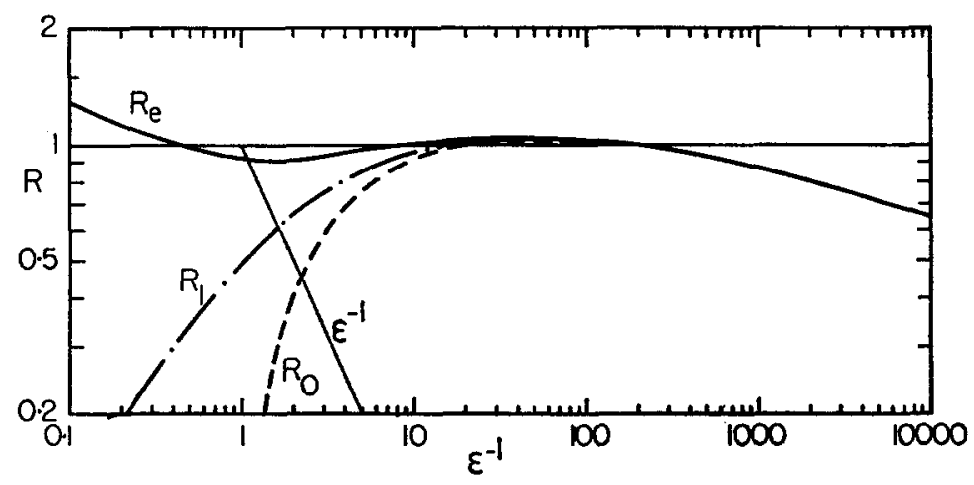

Fig. 1. Illustration showing the near $\varepsilon^{1 / 4}$ dependence of a logarithmic function in the range $5<\varepsilon<500$. Note expanded vertical scale which exaggerates departures from unity. Also shown is $\varepsilon^{-1}$.

that it is reasonable to approximate $\varepsilon^{-1} \ln \varepsilon$ by an asymptotic dependence such as $\varepsilon^{-\Omega}$ with $\Omega \sim \frac{s}{4}$. With this analytic form one can unify all excitation cross sections asymptotically into a convenient power law dependence. Using $W \sim 10 \mathrm{eV}$ as representative, we note that by 'asymptotic' we generally mean the domain of energies above, say, $500 \mathrm{eV}$ (e.g. $\varepsilon>50$ ). We must recognize, however, that at very high energies (e.g. $\varepsilon>10^{3}$ ), the logarithmic factor curves somewhat on log$\log$ paper. However, this is near the domain where relativistic effects come into play so that the usual BBA itself breaks down.

The usual BBA breaks down more seriously at low energies $\varepsilon \ll 50$, and, in the absence of theoretical models, it has been necessary to impose some phenomenological modification upon either the BBA or the simple power approximation of it. In view of the particular importance of the low energy domain to ionospheric problems, we shall describe a simple method of fitting the high energy behavior of a cross section and then quickly exploring a variety of low energy forms to establish the parameters in an analytic representation.

Here we first plot the cross section on convenient log-log paper, such as standard $3 \times 5$ cycle paper with $\sigma$ as ordinates and $E$ along the abscissa. For the vertical scale we use a standard unit of cross section $\sigma_{0}$ which is usually taken to be $10^{-16} \mathrm{~cm}^{2}$ or $1 A^{2}$. One next fits the high energy experimental data by an asymptotic 
line as is illustrated in Fig. 2. One can now quiokly obtain $A$ and $\Omega$ by using the asymptotic characterization

$$
\sigma \rightarrow\left(q_{0} A_{j} / W_{j}^{2}\right)\left(W_{j} / E\right)^{\Omega} .
$$

One first determines the parameter $\Omega$ by obtaining the ratio of the line as vertical to horizontal distances. Then one evaluates $\sigma$ at some convenient energy (say $E=100 \mathrm{eV}$ ). Then since $W_{j}$ 's are known, we can easily solve for the $A_{j}$.

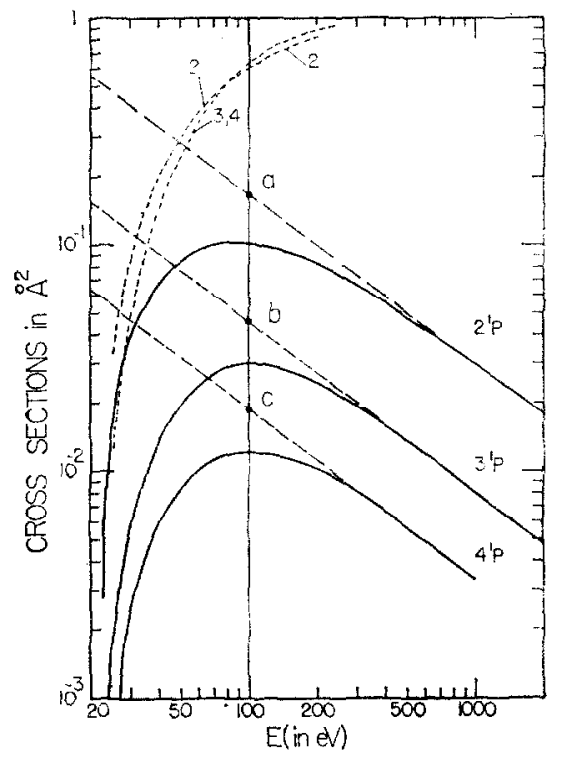

Fig. 2. Illustrations of allowed excitation curves. The solid line represents recent data of MoConkey et al. (1971). The long dash lines represents $E^{-8 / 4}$ extrapolations. The points $a, b$ and $c$ are used in the determination of the $A$ in equation (4).

The ratio data are shown by the short dashed lines.

Figure 2 illustrates the procedure using recent cross sections for the excitation of the $2^{1} P, 3^{1} P$ and $4^{1} P$ states of He (MoCoNKEY et al., 1971). In this instance we can approximately characterize all three curves with an $E^{-3 / 4}$ dependence as shown. From points $a, b$, and $c$ one can immediately evaluate the parameter $A(2)=37 \cdot 35$, $A(3)=10 \cdot 21$ and $A(4)=4 \cdot 22$.

Having established the parameters of the asymptotic line, one can determine graphioally the ratio of data points to the asymptotic line. This can be quiokly replotted on the same piece of graph paper by mechanical means with the aid of a divider or more simply with pencil and the edge of a piece of paper. These low energy ratio data which asymptotically should become unity are now the concern of the fitting procedure.

To proceed further, we must select a low energy modifier (LEM) of the asymptotic funotion $\varepsilon^{-\Omega}$. The LEM of Green and Barth is given by equation (2). Figure 3 shows a series of $\Phi_{I}(\varepsilon)$ expressed for various values of $\nu$. One sees that a reasonable variety of energy shapes is possible. 
Despite its versatility, the Green-Barth form may not be sufficiently flexible to cover all situations. Furthermore, this form is only really convenient for small integral values of $v$ and (to a much lesser extent) small half integral values of $v$.

In particular, if one wishes to fold these cross sections into a reciprocal loss function of the form (GREEN and STOLARSKI, 1966)

$$
L(E)^{-1}=L_{0}^{-1} \Sigma\left(E / E_{j}\right)^{\Omega_{j}}
$$

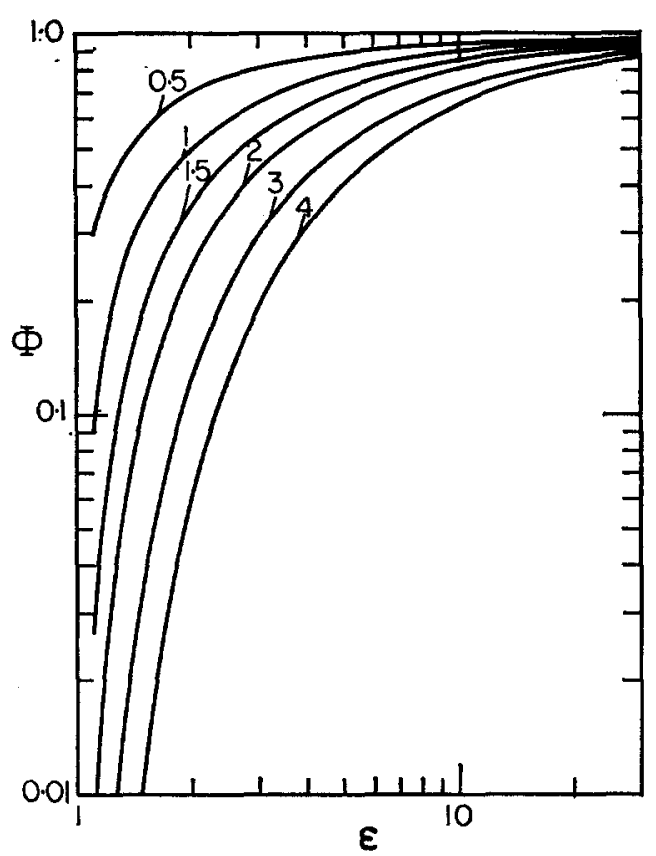

Fig. 3. LEM based upon $\left[1-\varepsilon^{-1}\right]^{\text {y }}$ for various values of $\boldsymbol{v}$.

where $L_{0}$ is a unit loss function and $E_{j}$ and $\Omega_{j}$ are parameters, it is more convenient to use an alternate LEM

$$
\Phi_{I I}=1-\varepsilon^{-\gamma} \text {. }
$$

Figure 4 illustrates the variety of shapes for various $\gamma$.

One can combine $\Phi_{I}$ and $\Phi_{I I}$ into a single composite LEM of the form

$$
\Phi_{\text {III }}=\left[1-\varepsilon^{-\gamma}\right]^{\nu}
$$

which is capable of many shapes determined by the two parameters $\gamma$ and $\nu$. It is mathematically convenient, however, only for small integral values of $v$. Figure 5 shows LEM's for $v=2$ and several values of $\gamma$.

In case the functional forms given above are not sufficiently broad to encompass observed cross sections, one can generalize these functions to three parameter forms such as

$$
\Phi_{\mathrm{IV}}=\frac{\left[1-\varepsilon^{-1}\right]^{y}}{[1+(\alpha / \varepsilon)]^{\mu}}
$$




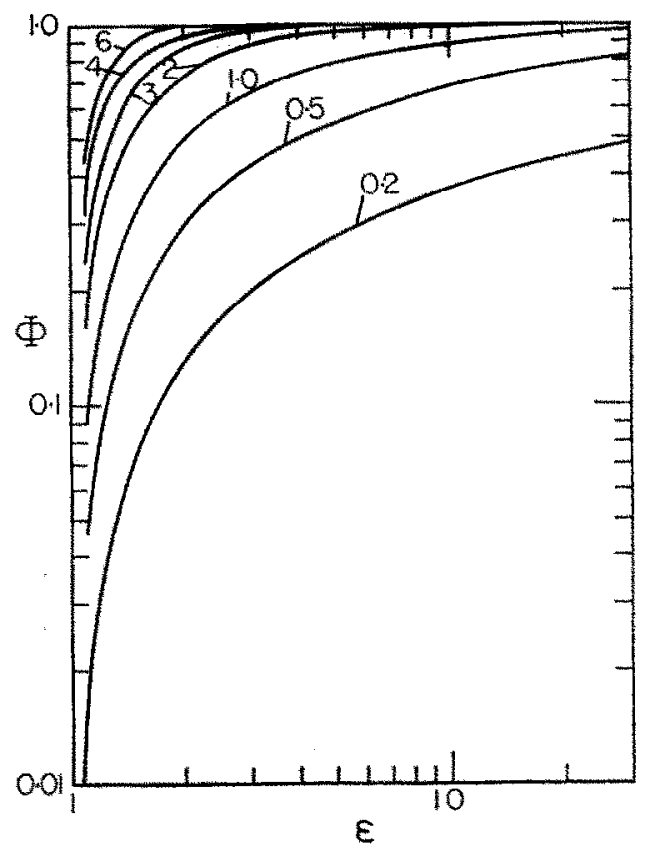

Fig. 4. LEM based upon $\left[1-\varepsilon^{-\gamma}\right]$ for various values of $\gamma$.

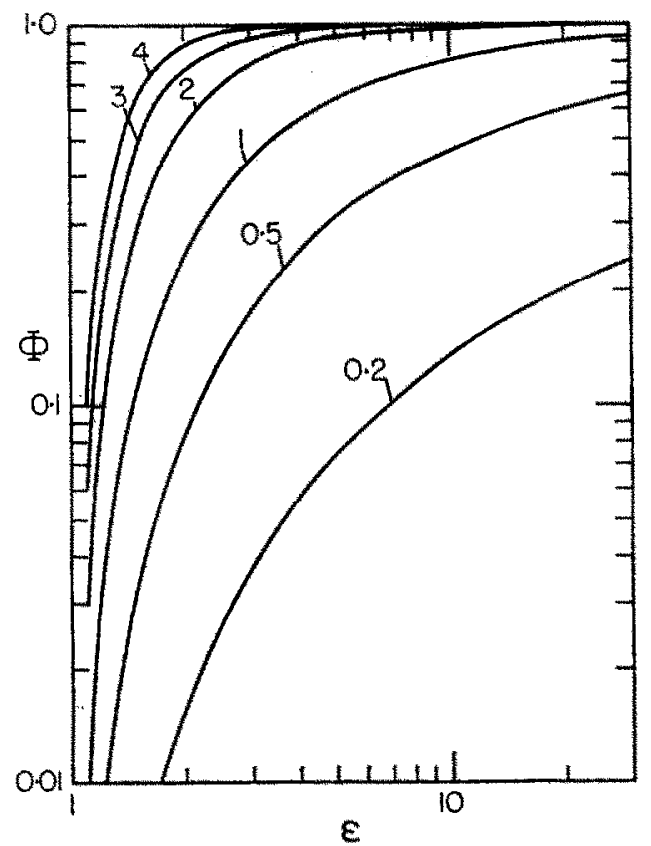

Fig. 5. LEM based upon $\left[1-\varepsilon^{-\gamma}\right]^{\nu}$ for $\nu=2$ for various values of $\gamma$. 
Figure 6 illustrates the case $\Phi_{\mathrm{IV}}$ for $\nu=1$ and $\mu=1$ for the various values of $\alpha$ shown. Experience has shown that finite values of $\alpha$ provide a versatile way of 'shaping' the middle energy region when the two or one parameter forms just do not fall in line.

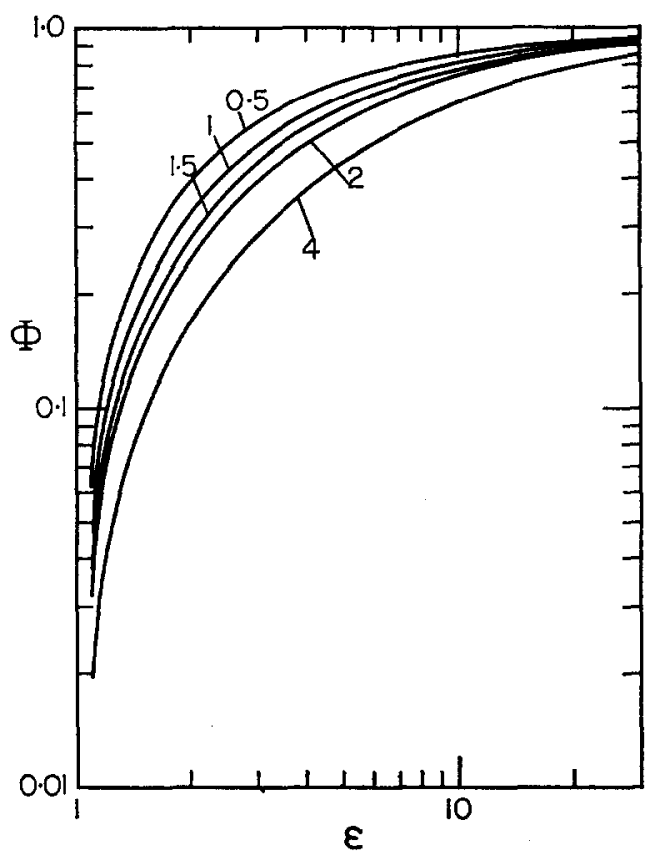

Fig. 6. LEM based upon $\left(1-\varepsilon^{-1}\right)^{\gamma} /[1+(\alpha / \varepsilon)]^{\mu}$ for the case $\nu=1, \mu=1$ and various values of $\alpha$.

As to the practical matter of evaluating the parameters, once a natural shape has been selected, one has a variety of choices.

These graphical procedures establish approximate parameters $\Omega, A$ and the LEM parameters to a degree of accuracy which may be adequate for most work. One can, however, refine these parameters by numerical fitting using recent non-linear least square (NLLS) computer programs.

One can generalize our analytic cross sections in a variety of ways. These more general forms can usually be fitted by a NLLS routine after approximate parameter estimates have been made with the special cases which we have discussed.

For cases when data is available over a very extended range of energies, say up to $50 \mathrm{keV}$, we may wish to give up the $E^{-\Omega}$ approximation in favor of one which goes over to $E^{-1} \ln E$. In such cases we may sometimes achieve an excellent fit all the way from threshold by the four parameter $(A, \nu, \alpha, \beta)$ function equivalent to that of GREEN and STRICKLAND (1971)

$$
\sigma=\left[q_{0} A / W^{2}\right] \psi, \quad \psi=[v(\varepsilon+\alpha)]^{-1} \ln \left[\beta(\varepsilon-1)^{\nu}+1\right] .
$$

In actuality the special case $\nu=1$ and $\alpha=0$, which is illustrated in Fig. 7, is fairly 


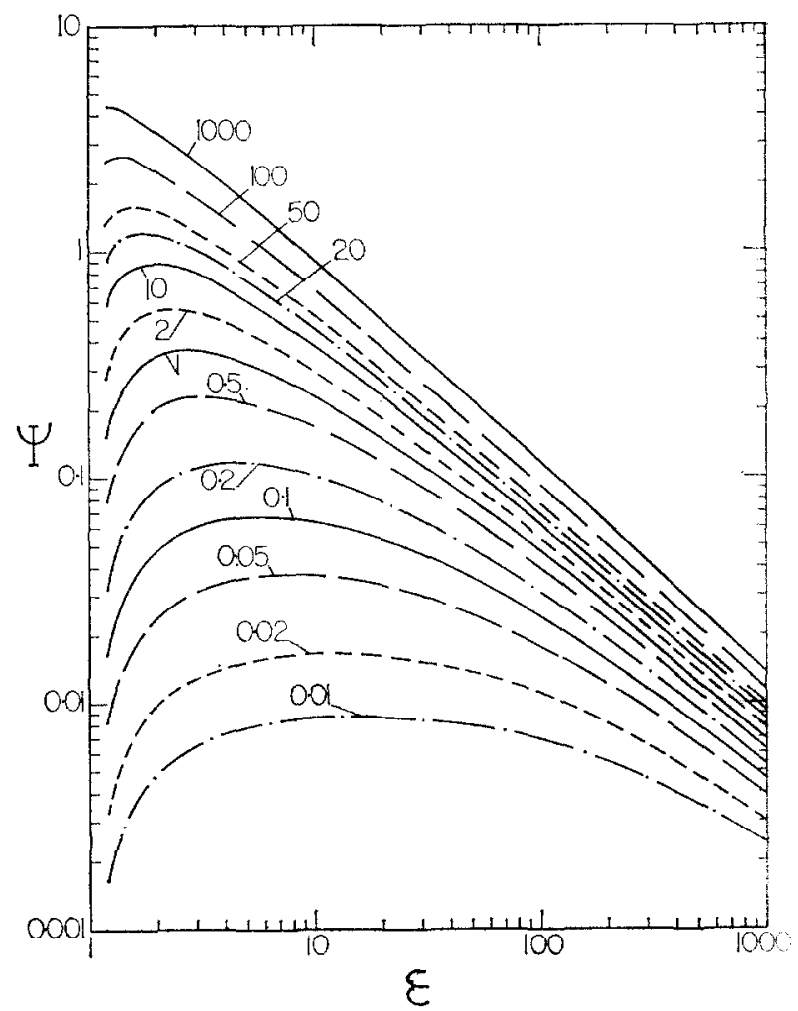

Fig. 7. The function $y$ for $y=1, \alpha=0$ for various values of $\beta$.

versatile. It is far better than

$$
\sigma=\left[q_{0} f_{n} / W^{2}\right] \psi, \quad \psi=\varepsilon^{-1} \ln \beta \varepsilon
$$

where $\beta=4 C_{n} W / R_{e}$ which is frequently used in the literature as an approximation which asymptotically goes over to the BBA. Since equation (9) can only be used with a computer anyway, one need only provide a reasonable set of guesses for a NLLS fitting routine. Here if the parameters $A$ and $\beta$ are known, we can use $v=1$ and $\alpha=1$ as guesses and then do an NLLS search for the optimum parameters. Alternatively one can graphically evaluate $A$ and $\beta$ by the use of Fig. 7 in relation to the data and then use a search routine to determine all four parameters.

Let us now turn our attention to the specific aspects of $\mathrm{N}_{2}, \mathrm{O}_{2}$ and $\mathrm{O}$ excitation cross sections.

\section{Excitation Cross Sechions}

(A) Allowed states of $\mathrm{N}_{2}, \mathrm{O}_{2}$ and $\mathrm{O}$

Allowed cross sections are those for states whose excitation from the ground state is permitted by electric dipole selection rules. Their characteristic shape with 
energy is very broad with a maximum at about 5-7 times threshold and a slow falloff as $E^{-1} \log E$.

The major allowed transition in $\mathrm{N}_{2}$ is at $12.85 \mathrm{eV}$ energy. This large peak in the impact spectrum was first observed by LASSETTRE (1964) and is composed mainly of the $b^{1} \pi_{\mu}$ state and the $n=3$ member of the Rydberg series leading to the ground state $X^{2} \Sigma_{0}{ }^{+}$of $\mathrm{N}_{2}{ }^{+}$. Another peak in the impact spectrum of $\mathrm{N}_{2}$ occurs at $14 \cdot 0 \mathrm{eV}$ and is composed of a mixture of the $p^{1} \Sigma_{u}+, b^{1} \Sigma_{u}+$ and probably many other states.

In $\mathrm{O}_{2}$ the $B^{3} \Sigma_{u}$ - state which leads to dissociation into $\mathrm{O}\left({ }^{2} P\right)+O\left({ }^{1} D\right)$ is allowed and corresponds to the impact spectrum peak observed at $8.4 \mathrm{eV}$ (LASSETTRE et al., 1964). Also observed is a peak at $9 \cdot 9 \mathrm{eV}$ which is almost cortainly duc to an allowed excitation. Figure 8 illustrates allowed and forbidden transitions for $\mathrm{N}_{2}$. Table 1 gives the parameters adopted for these states by Peterson et al. (1969).

\section{(B) Forbidden transitions}

Forbidden transitions are those whose excitation from the ground state violate electric dipole selection rules. Included are all excitations involving a change in spin multiplicity. These transitions proceed almost entirely by electron exchange and

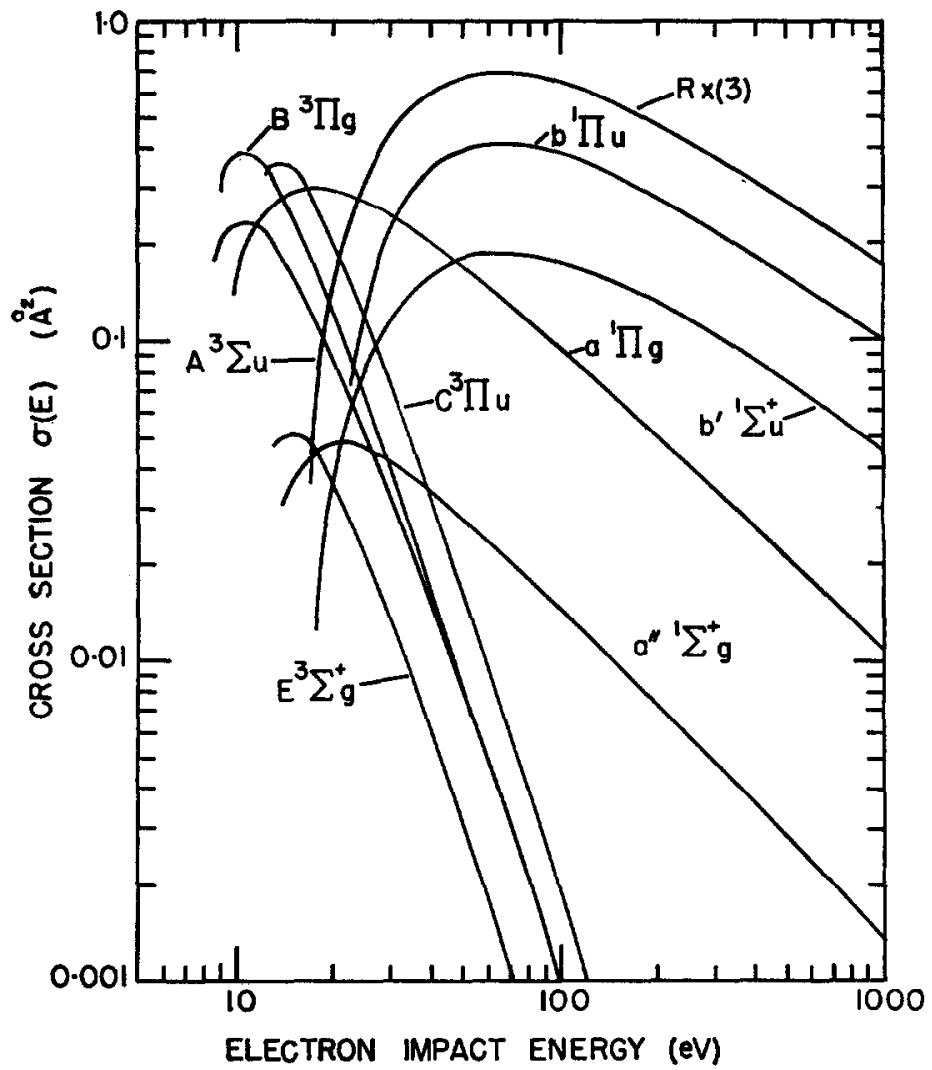

Fig. 8. Several excitation cross sections in $\mathrm{N}_{2}$ according to survey of GREEN et al. (1971). 
A. E. S. Green and R. S. Stolargri

Table 1. Excitation cross section parameters

\begin{tabular}{|c|c|c|c|c|c|c|}
\hline & State & $W$ & $A$ & $\Omega$ & $\nu$ & $y$ \\
\hline \multirow[t]{7}{*}{$\mathrm{N}_{2}$} & $A^{3} \Sigma_{u}^{+}$ & $6 \cdot 14$ & 0.226 & $3 \cdot 0$ & $\ldots$ & 1.0 \\
\hline & $B^{3} \pi_{g}$ & $7 \cdot 30$ & $0 \cdot 178$ & $3 \cdot 0$ & - & $3 \cdot 0$ \\
\hline & $C^{3} \pi_{u}$ & $11 \cdot 03$ & $0 \cdot 28$ & $3 \cdot 0$ & - & $3 \cdot 0$ \\
\hline & $a^{1} \pi_{\sigma}$ & $9 \cdot 10$ & $0 \cdot 136$ & $1 \cdot 0$ & - & $1 \cdot 0$ \\
\hline & $b^{1} \pi_{u}$ & $12 \cdot 85$ & 0.67 & 0.75 & 3 & - \\
\hline & $b^{\prime} \Sigma_{u}{ }^{+}$ & $14 \cdot 0$ & $0 \cdot 33$ & 0.75 & 3 & - \\
\hline & $\Sigma$ Rydberg & $13 \cdot 75$ & $2 \cdot 66$ & 0.75 & 3 & - \\
\hline \multirow[t]{6}{*}{$\mathrm{O}_{2}$} & $a^{1} \Delta_{q}$ & 0.98 & $0 \cdot 0005$ & $3 \cdot 0$ & - & $3 \cdot 0$ \\
\hline & $b^{1} \Sigma_{o}$ & $1 \cdot 64$ & 0.0005 & $3 \cdot 0$ & - & $3 \cdot 0$ \\
\hline & $A^{3} \Sigma_{u}+$ & $4 \cdot 5$ & 0.021 & 0.9 & - & $3 \cdot 0$ \\
\hline & $B^{3} \Sigma_{u}^{-}$ & $8 \cdot 4$ & $0 \cdot 23$ & $0 \cdot 75$ & $2 \cdot 0$ & - \\
\hline & $9.9 \mathrm{eV}$ allowed & $9 \cdot 9$ & 0.08 & 0.75 & $3 \cdot 0$ & - \\
\hline & $\Sigma$ Rydberg & $13 \cdot 5$ & $2 \cdot 77$ & 0.75 & $3 \cdot 0$ & - \\
\hline \multirow[t]{7}{*}{0} & $\mathbf{I}_{D}$ & 1.96 & 0.01 & 1.0 & $2 \cdot 0$ & - \\
\hline & $1_{g}$ & $4 \cdot 17$ & 0.0042 & $1 \cdot 0$ & - & 0.5 \\
\hline & $3_{S}$ & 9.53 & 0.0465 & $0 \cdot 75$ & $\mathbf{3} \cdot \mathbf{0}$ & - \\
\hline & 5 & $9 \cdot 15$ & 0.023 & $2 \cdot 0$ & - & $1 \cdot 0$ \\
\hline & $\tilde{\Sigma}(\Delta l=1, \Delta s=0)$ & $14 \cdot 2$ & 0.367 & 0.75 & $3 \cdot 0$ & - \\
\hline & $\Sigma(\Delta s=1)$ & $14 \cdot 7$ & $0 \cdot 694$ & $2 \cdot 0$ & $\ldots$ & $1 \cdot 0$ \\
\hline & $\Sigma(\Delta l=0, \Delta s=0)$ & $13 \cdot 5$ & 0.043 & 0.75 & $m$ & $2 \cdot 0$ \\
\hline
\end{tabular}

have characteristic cross sections which peak at less than two times the threshold energy and fall off very rapidly with increasing energy.

These characteristics are shown by the excitation cross sections for the triplet states in $\mathrm{N}_{2}$. These cross sections are usually measured by optical methods. Low energy electrons are used to excite the triplet states and the 2nd positive and 1st positive bands are observed. Franck-Condon factors are used to extrapolate to total excitation cross sections, and cascading contributions are used to extract cross sections for higher triplet states.

Table 1 shows our adopted parameters for the three major triplets in $\mathrm{N}_{2}\left(A^{3} \Sigma_{u}{ }^{+}\right.$, $B^{3} \pi_{g}$ and $C^{3} \pi_{u}$ ). A number of other triplet states have been observed in $\mathrm{N}_{2}$, but all are much smaller than the $A, B$ and $C$ states. These other triplets include the $E^{3} \Sigma_{g}^{+}, D^{3} \Sigma_{u}{ }^{+}, C^{\prime 3} \pi_{u}, B^{\prime 3} \Sigma_{u}$ and ${ }^{3} \Delta_{u}$ (see BURNs et al., 1969; MCConkEY and StMpson, 1969; Lassettre et al., 1969; Lassettre, 1970; Brinkmann and Trajmar, 1969; for measurements). We have not included these in our model set of parameters in Table 1, but anyone in need of a cross section for one or more of these states can take whatever data is available and use the methods described earlier in this paper to arrive at a set of parameters.

Also shown in Fig. 8 and Table 1 are the cross sections for the singlet forbidden cross sections of $\mathrm{N}_{2}$. These cross sections are identical to those appearing in GREEN et al. (1971) and Peterson et al. (1969). Note the broadness of the cross sections with energy. This is due to the fact that these cross sections do not have to proceed via an exohange meohanism. There is, however, always the possibility of a small exchange term which would put a sharp, low-energy peak on the cross section. 
The major spin change excitations in $\mathrm{O}_{2}$ are the $a^{1} \Delta_{g}$ and the $b^{1} \Sigma_{g}$ at $0.98 \mathrm{eV}$ and $1.6 \mathrm{eV}$ respectively. Data on these states are very sparse and are discussed by GREEN et al. (1970, 1971). The assumed parameters are in Table 1.

The other important forbidden excitation in $\mathrm{O}_{2}$ is the $A^{3} \Sigma_{u}+$ at $4.5 \mathrm{eV}$. For lack of any new information the cross sections given are the same as in PETERSON et al. (1969). The parameters are again in Table 1.

Our approach to the determination of cross sections for atomic oxygen is necessarily different from that for $\mathrm{N}_{2}$ and $\mathrm{O}_{2}$. Virtually no data exists on atomic oxygen and the cross sections must be determined entirely from rules developed from the study of other atoms. A set of rules of this type have been proposed by JusIck et al. (1967) from the study of $\mathrm{He}$ cross sections. These rules have also been discussed by PETERsow et al. (1969). The application of these rules to atomic oxygen has perhaps not been quite clear. Therefore in Table 1 we show a set of parameters for the most important transitions in 0 . These parameters are for either form $\left(\Phi_{\mathrm{I}}\right)$ or $\left(\Phi_{\mathrm{II}}\right)$ depending on whether $v$ or $\gamma$ is given. The determination of the energy levels and magnitudes of the higher members of the Rydberg series corresponding to each state given is described in the next section.

\section{(C) Autoionization and Rydberg states}

Above about $14 \mathrm{eV}$ in both $\mathrm{N}_{2}$ and $\mathrm{O}_{2}$, there are an exceedingly large number of states seemingly randomly oriented. These can be put in reasonable order by considering what happens to an electron as its principal quantum number increases. For high principal quantum numbers the molecular states exhibit a Rydberg-like character and can be described as states whose energy is proportional to

$$
W_{i}=I-\frac{R_{e}}{(n-\delta)^{2}}
$$

where $I$ is the ionization potential, $R_{\theta}$ is the Rydberg energy $(\approx 13.6 \mathrm{eV}$ ), and $\delta$ is a quantum defect. The transition probability to such states is proportional to $(n-8)^{-3}$.

Even though these Rydberg-like states may not be of specific importance to a particular problem, they must be included in any calculation which requires a total cross section or total energy loss rate. Many of these states also lie above the first ionization potential and hence lead to autoionization.

The cross sections from Rydberg states of $\mathrm{N}_{2}$ and $\mathrm{O}_{2}$ have deen determined by Stolarsi et al. (1967) and Watson et al. (1967). The method is to assume one Rydberg series leading to each ionization potential and to assume that the oscillator strengths in the series go as

$$
f_{o s}=\frac{f^{*}}{(n-\delta)^{3}}
$$

At the ionization potential the oscillator strength per unit energy interval in the continuum is then assumed to be equal to the limit of the series oseillator strengths 
per unit energy interval. This sets the magnitude parameter $f^{*}$ for the series. The quantum defeots for each series are determined by fitting the energy levels observed with equation (11). It has been tacitly assumed that the quantum defect is constant throughout a series and that the quantum defect defined in the energy equation is the same as that for the oscillator strength of a transition. The first of these assumptions is borne out by fit of the energy levels to the $E_{n}$ formula. The second of these assumptions is verified approximately by GANAS and GREEN (1971). In all eases the Rydberg series cross sections have been assumed to have the shape of allowed cross sections. Table III of Peterson et al. (1969) gives the parameters for the Rydberg series cross sections using the form

$$
\sigma_{j}=\frac{q_{o} f_{o j} C_{o}}{W_{j}^{2}}\left(1-\frac{W_{j}}{E}\right)^{p}\left(\frac{W_{j}}{E}\right)^{\Omega}
$$

with $\nu=3$ and $\Omega=0 \cdot 75$. The higher state cross sections are then determined as described above.

The contributions of these states to autoionization must then be determined by defining a branching ratio for each state whose energy is in the ionization continuum. These branching ratios were assumed to all be equal to 0.5 by STOLARSKI $e t$ al. (1967) and WATson et al. (1967) from inspection of photoionization data. Since much more data is now available, a further investigation should be made. The previously assumed branching ratio of 0.5 leads to autoionization of less than 5 per cent of the total ionization in $\mathrm{N}_{2}$ but close to 30 per cent in $\mathrm{O}_{2}$.

Our excitation cross sections have not considered the decomposition of electronic states into their vibrational components. These can be carried out by the approximate techniques based upon Franck-Condon factors (BarTH, 1966).

\section{Dissociative Excitation and Ionizamion}

Recent measurements (MUMMA, 1970; AJELlo, 1969) and analyses indicate that the processes of dissociation, dissociative excitation and dissociative ionization play a more important role in electron energy loss processes than had been suspected 5 or so years ago. For example, Prasad and Green (1971) show that dissociative excitation of $\mathrm{N}_{2}$ is probably the major process in the exaitation of many significant atmospheric emissions, e.g. the $1200 \AA$ radiation from NI. Grares et al. (1971) find that these processes are of major importance in energy deposition in $\mathrm{H}_{2} \mathrm{O}$. It would appear now that some of the oscillator strengths assigned on the basis of sum rules in earlier studies of $\mathrm{N}_{2}$ and $\mathrm{O}_{2}$ should be redistributed to these dissociative processes. GREEN et al. (1972) have compiled a set of parameters for equations (1) and (2) or equations (1) and (7) to approximately characterize such dissociative processes. In such calculations it is particularly essential to allow for the kinetic energy of the outgoing fragments as a part of the loss process. For dissociative excitation this has been accomplished by replacing $W_{3}$ by $\left(W_{f}+\bar{T}\right)$ where $T$ is an average kinetic energy.

In this same connection it might be remarked that one can use the same device to allow for vibrational energy of the excited species in molecular excitation processes. These facets of the energy deposition problem require much further study. 


\section{Low Energy Loss Mechanisms}

Many ionospheric calculations require a detailed knowledge of the loss processes for electrons at very low energies of a few $\mathrm{eV}$ and below. This subject has been reviewed by DaLGaRNo (1969), and thus we will just briefly mention the important processes. The most important processes to be considered are: (1) vibrational excitation of $\mathrm{N}_{2}$ and $\mathrm{O}_{2} ;$ (2) rotational excitation of $\mathrm{N}_{2}$ and $\mathrm{O}_{2} ;$ (3) electronic excitation of $\mathrm{O}_{2} a^{1} \Delta_{g}, b^{1} \Sigma$ and $O^{1} D ;(4)$ excitation of $O^{3} P$ fine structure levels; and (5) electron-electron losses. Some of these processes do not lend themselves quite as easily as others to the type of modeling we have been discussing. Vibrational excitation of $\mathrm{N}_{2}$ is a resonance process in which an intermediate $\mathrm{N}_{2}-$ is formed. The cross section has a very sharp peak at about $2 \mathrm{eV}$ and is extremely difficult to fit with the present models. In the paper by SToLanski et al. (1967), the total cross section was fit to the models given above. The fit was rather crude and did not include the effects of different energy losses for different vibrational states. Reasonably detailed cross sections have been calculated by CHEN (1964) and measured by ScHULz (1962) and others with good agreement.

Rotational excitation data is much more scarce and has never been included in our previous modeling. The subject is reviewed by DalaARno (1969), however. Electronic excitations of low-lying states have been discussed above.

The fine structure excitation of $\mathrm{O}^{3} P$ is a major cooling mechanism and has been discussed by DaLGarno and DegGes (1968) based on the cross sections of BRIEG and IIN (1966). The radiative transfer of the emitted 6300 radiation and its effect on cooling has been discussed by Kockarts and Peetermans (1970).

Photoelectron energy loss due to collisions with ambient electrons becomes relatively more important with increasing altitude due to the increased ratio of electron to neutral density. This loss is particularly important at energies below $10 \mathrm{eV}$. Most ionospheric calculations have made use of a simple expression for the loss rate for electron-electron collisions given by DaLgarno et al. (1964) which was based on the calculations of BUTLER and Buckingham (1961). SchUnK and HaYs (1971) have recently pointed out that the losses estimated by the form of Butler and Buckingham's results fit by Dalgarno are too small by as much as 50 per cent due to the neglect of quantum effects and the generation of plasma waves. Schunk and Hays expressions are very slightly density dependent, but within the range of ionospheric densities this is at most a 10 per cent effect. Their loss functions lend themselves very well to the type of modeling procedure we have described (GREEN and Strickland, 1970; Stolarsir, 1968; Swartz et al., 1971).

\section{Discussion and Conclusions}

Our object here has primarily been to provide the reader with a representative set of $\mathrm{N}_{2}, \mathrm{O}_{2}$ and $\mathrm{O}$ excitation cross sections and to refer him to sources of more detailed information. At the same time we call attention to the fact that theoretical and experimental cross sections results are still in a rapid state of flux and that almost any compilation will go out of date quite quickly. For this reason we believe every user should be equipped to some degree to re-adjust parameters for his input cross sections. The techniques and analytic forms described in Section 2 should 
handle most situations. In some instances one encounters a composite curve consisting of a slowly varying form at high energies and a rapidly varying form at low energies. In such instances one can usually fit the cross section by a linear combination of our analytic forms. Here one first subtracts away the slowly variating part and treats the residual in the same way as a simple curve. Sometimes such curve fitting techniques, when applied to rough experimental data, involve some degree of arbitrariness. This, however, is largely a reflection of the state of experimental knowledge. As the error bars become smaller the degree of arbitrariness, of course, is reduced.

Finally, it must be emphasized that this paper is neither a theoretical nor experimental work in atomic and molecular cross sections. Instead it is intended to serve as a compact representation of such works for the purposes of a third fraternity of aeronomical users of such cross sections. The analytic forms essentially serve as a compact means of communioations. If every cross section involved in aeronomical applications were presented simply as data, we would quiokly exhaust all library shelf space or computer storage space. Analytic forms which are good over broad energy ranges can provide compact and accurate means of communicating information, and this, in the last analysis, may be their most valuable purpose.

Acknowledgements-The authors would like to thank Drs. L. R. Prtenson and S. S. Prasad for permission to quote as yet unpublished results.

\section{References}

Arrexo J. M.

Barth C. A.

Borst W. L. and ZIPF E. C.

BrIEG E. L. and LIN C. C.

Brentracank R. T. and Trajmar S.

Burns D. J., Bnmpon F. R, and MaConkex J. W.

Buther S. T. and Buckmanay M. J. 1962

Crermak V.

Chrow J. C. Y.

Dalaakino A., Moelrox M. B. and Morrirti R. J.

Dalgaraxo A. and Dergare T. C.

Dalaario A.

Ganas P. S. and Green A. E. S.

Greme A. E. S, and Barth C. A.

GriegN A. E. S. and Stolariski R. S.

Grams A. E. S. and Stritoruand D.J.

Graes A. E. B., Ouvero J. J. and Stagax R. W.

Hifrzberara $G$.

1970

1966

1970

1969

1966

1964

1804

1968

1969

1971

1965

1866

1870

1971

1950
1970 J. chem. Phys. 53, 1156.

1966 The Middle Ultraviolet: Its Science and Technology (Edited by A. E. S. GrEEN). p. 177. Wiley, New York.

Phys. Rev. A1, 834.

Phys. Rev. 161, 67

Annls G6ophys. 86, 201.

J. Phys. B, 2, 52.

Phys. Rev. 128, 1.

J. chem. Phys. 44, 1318.

Atomic Collision Processes, p. 428. North. Holland, Amsterdam.

Planet. Space Sci. 11, 463.

Planet. Space Sci. 16, 125.

Can. J. Chem. 47, 1723.

Phys. Rev. A4, 192.

J. geophys. Res. 70, 1083.

The Middle Ultra violet: Its Science and Technology (Edited by A. E. 8. Graise), p. 165. Wiley, New York.

J. Franklin Inst. 990, 227.

Biophysical Aspects of Radiation Quality, p. 79. IAEA, Vienna.

Molecular Spectra and Moleoular Structure I. Spectra of Diatomic Molecules. Van Nostrand, Princeton, N.J. 
Jusick A. T., Watron C. E., Pethrson 1967 L. R. and Grenen A. E. S.

Krmatrear L. J. and DUNA G. H.

Kockarts G. and Pehrehrmans W.

LASSFTTRRE E. N.

LAsgirtTRE E. N. et al.

LASSHFTRE E. N. et al.

LASSEFTTRE E. $\mathbf{N}$.

MCCoNKEYY J. W. and SnMpson F. R.

MoConkey J. W., Donaldoson F. G. and HENDER M. A.

MotT N. F. and Massey H. S. W.

Peterson L. R., Prasad S. S. and Griben A. E. S.

Prabad S. S. and Greete A. E. S.

ScHULz G. J.

SoHUNK R. W. and Hays P. B.

Stolarzkr R. S., Dulock V. A., JR., Watson C. E. and Green A. E. S.

SWARTZ W. E., NI8BET J. S. and Grehen A. E. S.

WAtgon C. E., DULOOK V. A., JB., Stolargit R. S. and GreerN A. E. S.
J. geophys. Res. 72, 15.

1966 Rev. Mod. Phys. 38, 1.

1970 Planet. Space Sci. 18, 271.

1969 Can.J. Chem. 47, 1733.

$1964 J$. chem. Phys. 40, 1208 1218, 1222, 1232, $1242,1248,1256,1271$.

1959 Radiation Res. Suppl. 1, 530.

1969 Can.J. Chem. 47, 1733.

1969 J. Phys. B. 2, 923.

1971 Phys. Rev. Letts 26, 1413.

1965 The Theory of Atomic Collisions. Oxford. 1969 Can. J. Chem. 47, 1774.

1971 J. geophys. Res. 76, 2418.

1964 Phys. Rev. 135, A988.

1971 Planet. Space Sci. 19, 113.

$1967 J$. geophys. Res. 72, 3953.

$1972 J$. geophys. Res. (to be publishod).

1967 J. geophys. Res. 72, 3961.

Reference is also made to the following unpublished material:

Mumara M. J.

1970 Dissociative excitation of atmospherio gases, Ph.D. dissertation, University of Pittsburgh. 